\title{
Male age influence on male remating and progeny production in Drosophila malerkotliana
}

\author{
Shivakumar P., Krishna M. S.* \\ Drosophila Stock Center, Department of Studies in Zoology, University of Mysore, Manasagangotri, Mysore, Karnataka State, India \\ Email address: \\ drosokrish@gmail.com (Krishna M. S.), skumarpmys@gmail.com (Shivakumar P.) \\ To cite this article: \\ Shivakumar P., Krishna M. S.. Male Age Influence on Male Remating and Progeny Production in Drosophila Malerkotliana. American \\ Journal of Bioscience. Vol. 2, No. 3, 2014, pp. 95-101. doi: 10.11648/j.ajbio.20140203.13
}

\begin{abstract}
Reproductive success of the male is not only depends on his success in mating, but the number of females he could inseminate in a given time. The age effect on male remating and progeny production has been studied in Drosophila malerkotliana. It was found that middle aged males had a greater percentage of remating than those of young or old males. Male mated two females in an hour had copulated longer, laid a greater number of eggs and produced the greatest number of progeny than those males which mated with one female in an hour. This result found to be similar in all the male age classes studied. It was also noted that young male copulated longest in the experiment involving male mated only one female in 1 hour. Female that mated with middle aged male laid least fecundity in the experiment involving male mated only one female in 1 hour. Whereas female that mated with middle aged male had produced greater progeny in the experiment in which male mated with one/two females in 1 hour. Among the male mated with $1^{\text {st }}$ and $2^{\text {nd }}$ female in an hour male invested more in $1^{\text {st }}$ mated female compared to $2^{\text {nd }}$ mated female.
\end{abstract}

Keywords: Drosophila Malerkotliana, Male Age, Male Remating, Fertility

\section{Introduction}

All biological processes directly related to reproduction plays an important role in determining fitness. Collectively, these processes determine the fertility component of fitness, which fertility is defined in its broadest sense (Turner and Anderson 1983). This is because reproductive capacity is particularly a good index of fitness in organisms that go through repeated cycles of rapid population growth and it has evolved as a way for species to maximize their potential for survival [44].

The sexual behaviors of various species of Drosophila, with particular reference to their basic courtship patterns, genetic control, role of stimuli, and contributions of the 2 sexes to variations in mating. Repeated mating has been studied extensively [6,10,29].

Remating is common among many species of Drosophila under both natural and laboratory conditions. Multiple mating in Drosophila bears a direct relation to fitness [24]. Remating time of male and female in different species of Drosophila has been studied [31,39, 40,43]. Remating and sperm storage are specific features that can play important roles in determining female fecundity, male mating success. Remating reduces the maternal survival [26, 28,30].
Markow Quaid and Kerr [27] have found that females are able to distinguish between mated and unmated males and those that had a previous mating experience, showing preference for virgin males.

It has been widely demonstrated that males of different species of Drosophila can inseminate more than a single female, and the mating ability of males is influenced by genetic and other factors [32,34,36,37]. According to Gromko [12], multiple mating is widely believed to be advantageous for males, and selection of males can produce a correlated response in females.

A great diversity of male traits is known to influence female mating decision. Some of the factors that can determine male mating success include size, diet and early adult experience $[1,7,8,19,25]$. Male size in insects may affect reproductive activities including success in intrasexual encounters, sperm precedence and female fecundity. For many of these activities a larger size confers a reproductive advantage. One male characteristic that has received a lot of attention as a possible cue for female mating decision is age $[5,15,22,42,46]$. The effect of male age on reproductive success has been of great interest to evolutionary biologists.

The importance of male age on sexual behavior is not 
straightforward, and in most cases, only 1 or a few aspects of male fitness like success in inter-male competition, fecundity, fertility, and longevity have been studied $[2,33$, 42,]. In fact, remating is also a strategy for improving the fitness of individuals of polygamous populations. In Drosophila, the influence of female remating on fitness has been studied [41]. The further influence of male age on male remating ability has been largely ignored.

Therefore present study has been undertaken in $D$. malerkotliana. It is a cosmopolitan species is a member of the bipectinata complex of the ananassae subgroup [3-4]. It has a wide ecogeographical distribution ranging from India through South east Asia and New Guinea to Fiji and Samoa in the Pacific [4]. It is a common occurrence in the Indian subcontinent and has attracted the attention of various Indian workers who are using this species for past the few years. They have carried out extensive studies on population and behavior genetics of this species and have established the phylogenetic relationship between $D$. malerkotliana and other members of the bipetinata complex based on chromosome analysis, hybridization studies and isozyme analysis [17, 18, 20, 35, 45]. These findings provide interesting and important information concerning certain aspects of evolutionary genetics of this species. However, in this species, male age effect on male mating ability and progeny production has not been studied therefore the present study has been undertaken in $D$. malerkotliana. Hence the present investigation has been undertaken in D. malerkotliana to understand male age influence on the male mating ability and progeny production.

\section{Materials and Methods}

\subsection{Establishment of Experimental Stock}

Flies used in the present investigation were obtained from the experimental stock of $D$. malerkotliana established from progenies of fifty wild caught females (ISO-female line) collected at Chamundi Hills, Mysore, Karnataka state, India. In each generation fly were mixed together and redistributed to different culture bottles each with 20 males and 20 females. These flies were cultured using wheat cream agar media and maintained them at $22 \pm 1^{\circ} \mathrm{C}$ at $\mathrm{RH}$ of $70 \%$ of 12 hour light: 12 hour day cycle for three generations to allow them to acclimatize to laboratory conditions. At 4th generation, virgin females and unmated males were isolated within three hours of their eclosion and were aged as required for the experiment.

\subsection{Assigning Male Age Classes}

Before the start of the experiment male age classes were assigned by studying longevity of male $D$. malerkotliana. Unmated males were individually transferred into a vial containing wheat cream agar medium once a week and maintained them in above lab condition. This process was continued until their death and the longevity was recorded from the time of its eclosion until death in the days. A total of 50 replicates was made and mean longevity data calculated was found to be $60 \pm 2$ days. In addition to this, mating activities of males were also studied from day 1 st to $55^{\text {th }}$ day. Results showed that from 2 nd day to $54^{\text {th }}$ day male showed all the courtship activities and later it started to decline. Therefore, male age classes where we assigned 2-3 days for young, 24-25 day for middle aged and 46-47 days for older males.

The first set of flies emerged from cultured bottles were allowed to age for 46-47 days (to obtain old males). When these flies reached $25^{\text {th }}$ day the next set of new flies were isolated and allowed to age for 24-25 days (to obtain middle aged males). When the second set of flies reached $25^{\text {th }}$ day and the first set of flies reached $45^{\text {th }}$ day, then the new set of flies was isolated and was aged for 2-3day (to obtain young males). These young, middle and old aged males along with 5-6 day old virgin females obtained from the same culture and maintained them individually under uniform environmental conditions as described above. This procedure allowed us to grow the flies of all the three age classes in the same environmental condition and conduct the experiment at the same time.

\subsection{Male Age Influence on Percentage of Male Mating Ability}

To study male age effect on male remating. A 5-6 day old virgin female and a male (young/middle-aged/old) were aspirated into an Elens-Wattaiux mating chamber (ElensWattaiux, 1964) and observed for an hour. Flies which did not mate within an hour were discarded. If the flies mate, they were allowed to complete copulation and the duration of copulation was recorded (time between initiation to termination of copulation of each pair). Soon after mating the mated female was transferred to a vial containing wheat cream agar medium to check for larval activity. Mated male was once again provided with 5-6 days old virgin female and observation was continued up to an hour. If the second female that did not mate within the time was equally discarded. If mating occurred, the duration of copulation was recorded and mated female was transferred to a new vial to observe larval activity. A total of 50 trials was carried out separately for each of the three male age classes (young-50, middle aged-50, old male-50). The percentage of male remating ability was recorded.

\subsection{Male Age Influence on Male Mating Ability and Male Fitness}

Five to six days old virgin females and a male (young/middle aged and older) were aspirated into an Elens-Wattaiux mating chamber [9] and observed for an hour. Flies which did not mate within an hour were discarded. If the flies mated, they were allowed to complete copulation and duration of copulation was recorded (time between initiations to termination of copulation of each pair). Soon after mating mated female was transferred into 
a vial containing wheat cream agar medium every 24 hours. This was continued until the death of the female. Mated male was once again provided with 5-6 day virgin female and observation was continued up to an hour. If mating did not occur with second female within the time, then it was considered as male mated once in an hour. If mating occurred with the mated second female was allowed to complete copulation and the duration of copulation was recorded. Mated female was transferred to a vial containing wheat cream, medium once in 24 hours and was continued up to the death of the second female. The total number of eggs laid and the progeny produced by male mated with the first and second female was recorded. Fifty male mated with one female in an hour and mated with two females in an hour were separately used for each male age class (young-50, middle aged-50, old male-50). Data of male mated with second female were added together to calculate duration of copulation, fecundity and fertility. Duration of copulation, eggs laid and the progeny produced data by male mated with one female within 1 hour, male mated with two females within 1 hour. Male mated with first female and second female was subjected to two way ANOVA followed by Tukey's post hoc test.

\section{Results}

The percentage of males remating at young, middle and old age of the D. malerkotliana is provided in figure-1. It was noticed that middle aged males had the highest percentage of remating potential compared to the young and old males.

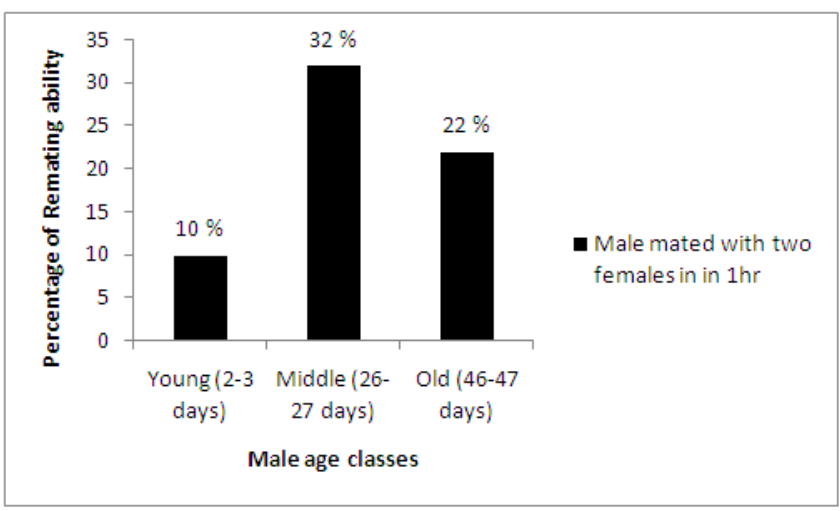

Figure 1. Percentage of mating success of male mated twice with female within one hour in D. malerkotliana

\subsection{Male age Influence on Copulation Duration}

Figure 2 a revealed that duration of copulation was found to be longer in male mated with two females in 1 hour than that those male mated with one female in 1 hour. This was found to be similar in all the male age classes. Among male age classes old male copulated longer than middle aged and young males. Two-way ANOVA followed by Tukey's posthoc test applied on above data showed significant variation in copulation duration between male age classes, between male mated with one female and two females in 1 hour [Table-1]. Tukey's post-hoc test showed that old male copulated significantly longer than middle aged and young male mated with one female in 1 hour. In contrast to this copulation duration of male mated with two females in 1 hour was found to be insignificant varied different between male age classes.

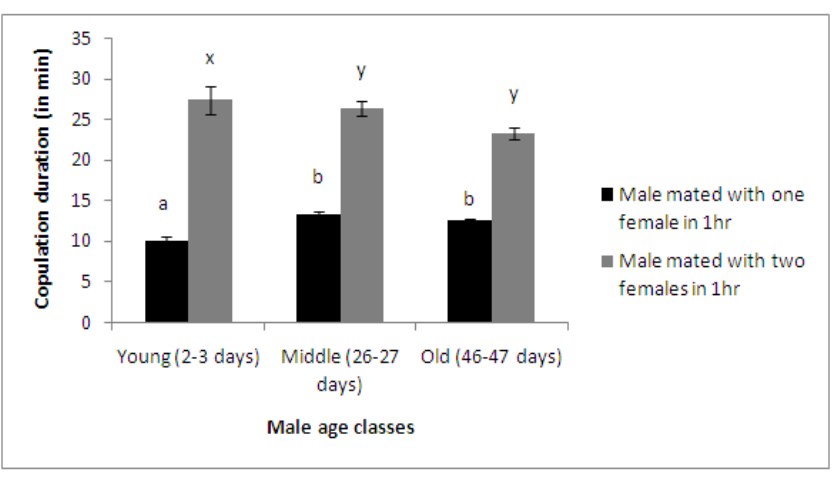

Figure 2a. Male age influence on Copulation duration (in min) of male mated with one female in 1 hour and two females in 1 hour in D. malerkotliana. (Values are Mean $\pm S E$ )

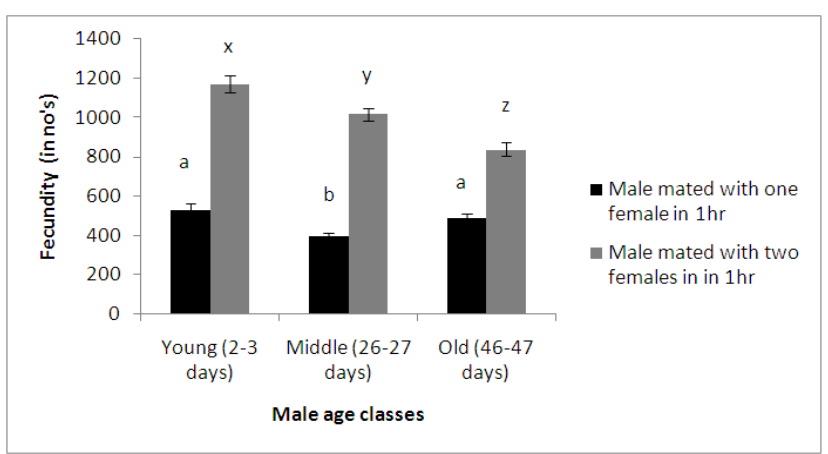

Figure 2b. Male age influence on Fecundity (in nos) of male mated with one female in 1 hour and two females in 1 hour in D. malerkotliana. (Values are Mean $\pm S E$ )

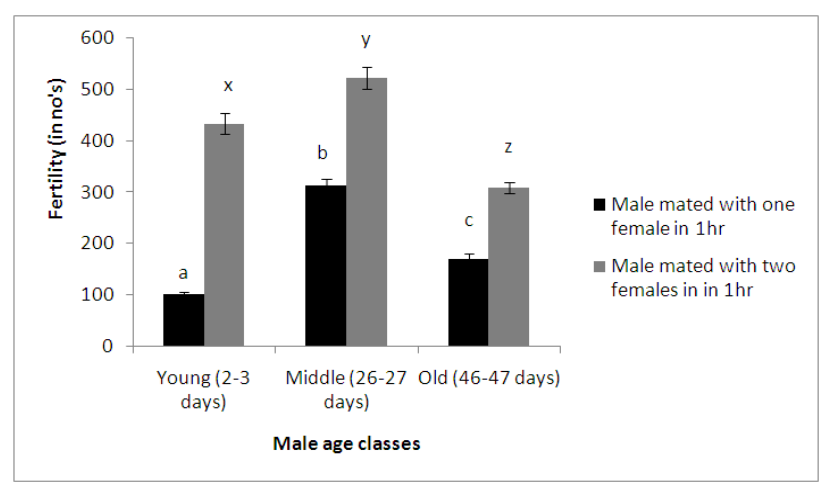

Figure 2c. Male age influence on Fertility (in nos) of male mated with one female in 1 hour and two females in 1 hour in D. malerkotliana. (Values are Mean $\pm S E$ ) 
Table 1. Two-way ANOVA of male age effect on fitness traits between male mated with one female and two females in D. malerkotliana

\begin{tabular}{|c|c|c|c|c|c|c|}
\hline Parameter & Source & Type III Sum of Squares & df & Mean Square & F-value & Sig \\
\hline \multirow{5}{*}{$\begin{array}{l}\text { Copulation } \\
\text { duration }\end{array}$} & Age & 464.007 & 2 & 232.003 & 5.458 & .005 \\
\hline & Mating status & 14145.333 & 1 & 14145.333 & 332.765 & .000 \\
\hline & Age * Mating status & 127.847 & 2 & 63.923 & 1.504 & $224^{\mathrm{Ns}}$ \\
\hline & Error & 12497.480 & 294 & 42.508 & & \\
\hline & Total & 134020.000 & 300 & & & \\
\hline \multirow{5}{*}{ Fecundity } & Age & 2108926.407 & 2 & 1054463.203 & 20.478 & .000 \\
\hline & Mating status & 14093301.763 & 1 & 14093301.763 & 273.700 & .000 \\
\hline & Age * Mating status & 787221.927 & 2 & 393610.963 & 7.644 & .001 \\
\hline & Error & 15138602.340 & 294 & 51491.845 & & \\
\hline & Total & 219852919.000 & 300 & & & \\
\hline \multirow{5}{*}{ Fertility } & Age & 932240.580 & 2 & 466120.290 & 46.697 & .000 \\
\hline & Mating status & 3323479.253 & 1 & 3323479.253 & 332.952 & .000 \\
\hline & Age * Mating status & 319439.687 & 2 & 159719.843 & 16.001 & .000 \\
\hline & Error & 2934668.760 & 294 & 9981.867 & & \\
\hline & Total & 37500766.000 & 300 & & & \\
\hline
\end{tabular}

Figure 3 a. Shows the duration of copulation of male mated with $1^{\text {st }}$ female and $2^{\text {nd }}$ female in 1 hour with young, middle-aged and older males. Duration of copulation with $1^{\text {st }}$ female was highest in middle aged males compared to that of young and old males while duration of copulation with $2^{\text {nd }}$ female was highest in young male and it was found to be more or less the same in middle aged and old males. Twoway ANOVA followed by Tukey's post-hoc test carried out on above data showed significant variation between male age classes and between $1^{\text {st }}$ female and $2^{\text {nd }}$ female. However insignificant variation was noticed in the interaction between male age and mating status (Table 2). Tukey's test showed that among male age classes, middle aged female mated with first female copulated longer compared to young or old male mated with first female (Fig-3a).

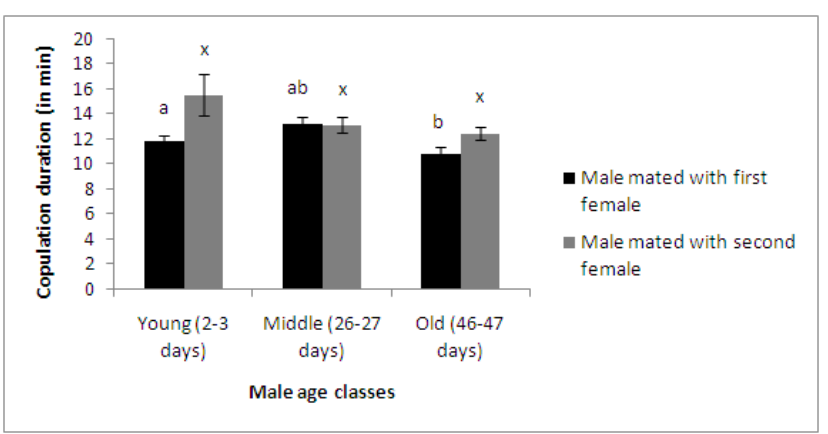

Figure 3a. Male age influence on Copulation duration (in min) of male mated with the 1 st female in 1 hour and $2^{\text {nd }}$ female in 1 hour in D. malerkotliana (Values are Mean \pm SE)

Table 2. Two-way Anova of male age effect on fitness traits between $1^{\text {st }}$ and $2^{\text {nd }}$ mated female in D. malerkotliana

\begin{tabular}{|c|c|c|c|c|c|c|}
\hline Parameter & Source & Type III Sum of Squares & df & Mean Square & F -value & Sig \\
\hline \multirow{5}{*}{$\begin{array}{l}\text { Copulation } \\
\text { duration }\end{array}$} & Age & 224.747 & 2 & 112.373 & 3.257 & .040 \\
\hline & Mating status & 225.333 & 1 & 225.333 & 6.531 & .011 \\
\hline & Age * Mating status & 173.307 & 2 & 86.653 & 2.512 & $083^{\mathrm{NS}}$ \\
\hline & Error & 10143.280 & 294 & 34.501 & & \\
\hline & Total & 60432.000 & 300 & & & \\
\hline \multirow{5}{*}{ Fecundity } & Age & 1367187.547 & 2 & 683593.773 & 17.478 & .000 \\
\hline & Mating status & 1487129.613 & 1 & 1487129.613 & 38.022 & .000 \\
\hline & Age * Mating status & 369029.547 & 2 & 184514.773 & 4.718 & .010 \\
\hline & Error & 11499055.880 & 294 & 39112.435 & & \\
\hline & Total & 90894950.000 & 300 & & & \\
\hline \multirow{5}{*}{ Fertility } & Age & 582202.087 & 2 & 291101.043 & 32.781 & .000 \\
\hline & Mating status & 13.230 & 1 & 13.230 & .001 & $969^{\mathrm{NS}}$ \\
\hline & Age * Mating status & 143953.380 & 2 & 71976.690 & 8.105 & .000 \\
\hline & Error & 2610786.220 & 294 & 8880.225 & & \\
\hline & Total & 16657409.000 & 300 & & & \\
\hline
\end{tabular}

\subsection{Male Age Influence on Fecundity}

The total number of eggs laid by male mated with one female (once mated) in 1 hour and two females (twice mated) in 1 hour is provided in the figure $-2 b$. In both the cases male mated with two females in 1 hour had laid the greatest number of eggs than males mated with one female in 1 hour. Among male age classes middle aged male, which mated with one female in 1 hour had lowest fecundity compared to the young or old, male, while fecundity of the 2nd female decreased with increasing age of the males. Two-way ANOVA followed by Tukey's posthoc test carried out on the above data showed significant variation in the total number of eggs laid between male age classes, between male mated with one female (once mated) in 1 hour and male mated with two females (twice mated) in 1 hour. Also, interaction between male mating status and male age classes. Tukey's post-hoc test showed that young male mated with one female in 1 hour had laid a significantly greater number of eggs compared to females 
that mated with middle-aged or old males. Similarly, middle aged male that mated with one female in 1 hour had laid a significantly greater number of eggs compared to the females that mated with old males by Tukey's test.

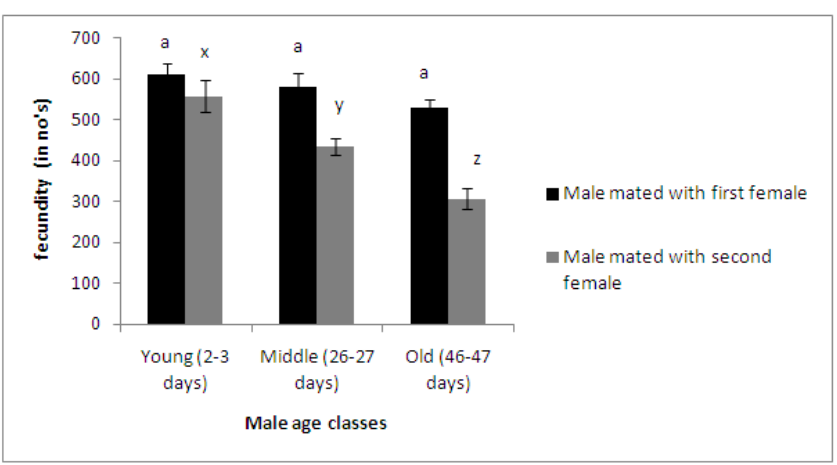

Figure 3b. Male age influence on Fecundity (in nos) of male mated with the 1 st female in 1 hour and $2^{\text {nd }}$ female in 1 hour in D. malerkotliana (Values are Mean $\pm S E$ )

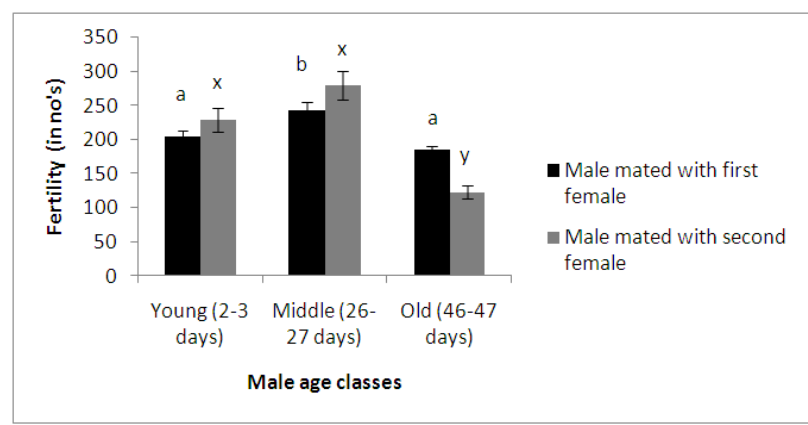

Figure 3c. Male age influence on Fertility (in nos) of male mated with the 1 st female in 1 hour and $2^{\text {nd }}$ female in 1 hour in D. malerkotliana (Values are Mean $\pm S E$ )

Figure- $3 \mathrm{~b}$. Shows the total number of eggs laid by the male mated with $1^{\text {st }}$ and $2^{\text {nd }}$ female. The number of eggs laid by first female mated was greater within that of than second mated female in all the three male age classes studied. Two-way ANOVA followed by Tukey's post-hoc test applied on above fecundity showed significant variation between male age classes, between $1^{\text {st }}$ mated and $2^{\text {nd }}$ mated females and also the interaction between male age and mating status. Tukey's post hoc test showed that among male age classes young male mated with first female had significantly greater number of eggs compared to the middle aged or old aged males mated with first female.

\subsection{Male Age Influence on Fertility (Progeny Production)}

Figure-2c. Shows the total number of progeny obtained from male mated with one female in 1 hour and male mated with two female in 1 hour. It was noticed that females mated with middle aged male had a greater number of progeny than females mated with old or young males. Furthermore, male mated with two females in 1 hour had greater progeny than male mated with one female in 1 hour. Two-way ANOVA followed by Tukey's post-hoc test applied on fertility data showed significant variation among male age classes, between male mated with one female in one hour and two females in one hour.

Results in Figure-3c. Shows that, the number of progeny obtained from male mated with $2^{\text {nd }}$ female was higher than the number of progeny obtained from the male mated with the 1st female in all the three age classes studied. Two-way ANOVA followed by Tukey's post-hoc test on the above data showed significant variation between male age classes and also between the male mated with the $1^{\text {st }}$ and $2^{\text {nd }}$ mated female. Whereas in contrast to this, the interaction between mating status and male age classes showed insignificant variation.

\section{Discussion}

Age in male insects may affect the reproductive activities including success in intra sexual counters, sperm precedence and female fecundity etc. [42]. However, male reproductive success depends on the number of females inseminated by a male in a given unit of time. Therefore, male remating ability forms one of the strategies to improve male fitness [23]. Males of all the three age classes of D. malerkotliana remate within an hour suggesting that ability to inseminate more than one female becomes the inherent ability of male to increase his fitness. Singh and Singh [38] studied D. ananassae and noticed differences in male remating ability among different geographical populations. Guruprasd [14] also studied D. bipectinata are found differences in male remating in four geographical populations.

In the present study in D. malerkotliana middle aged males have a greater percentage of remating than young and old males. This suggests that the age has significant influence on the percentage of male remating ability (Fig 1). The greater percentage of remating ability of middle aged male could be attributed to the occurrence of age specific variation in male fitness. In our study it was also noticed that in all the three male age classes all the males have not remated and this varies with different male age classes. This raises the question that if male remating indeed increases male fitness, then all the males have to undergo remating. In the present study copulation duration and progeny production of male mated with one female in 1 hour and male mated with two females in lhour were assessed to rule out the implication of male remating in $D$. malerkotliana.

Figure It was observed in this study that male mated with two females in an hour had copulated longer and laid a significantly greater number of eggs and progeny compared to male mated with one female in an hour (Figure.2a and table 1). This suggests that male remating is really advantageous and this enhances fitness. The result of our study also conforms to the result obtained from [12] where it was observed that multiple mating in D. melanogaster was advantageous to males and selection of males can produce a correlated response in females. In our study it 
was equally observed that young, middle aged and old males D. malerkotliana remated males had greater productivity. Our study conforms to the study of [38] in Drosophila ananassae. Furthermore, the present study is in consonance with [23] on D. malerkotliana and D. bipectinata. Thus, it was noted that in species of Drosophila male remating ability is not only influenced by genetic and environmental factors.

Figure. 3 and Table 2 reveals that male investment to first and second mated females in 1 hour varies. Copulation duration of male with $1^{\text {st }}$ female was found to be more or less the same. While male mated with $2^{\text {nd }}$ female, the middle aged male copulated longer compared to old or young male that copulated with $2^{\text {nd }}$ female. In contrast to this the number of eggs laid by male mated with first female was significantly greater compared to the male mated with $2^{\text {nd }}$ female. This suggest male invest more in the $1^{\text {st }}$ female he encountered in 1 hour.

From the results it can be seen that all age classes of males had inseminated and invested more towards fecundity in the $1^{\text {st }}$ female than in the $2^{\text {nd }}$ female that had mated. This result was found to be similar in males of all age groups, suggesting males to have invested more in $1^{\text {st }}$ female than $2^{\text {nd }}$ female. Our results confirm the work of Singh and Singh [28] in D. ananassae and Guruprasad [14] in D.bipectinata. From our results and other studies it can be demonstrated that older males increase their fitness by remating effectively with a number of individuals. At the same time make effective investment in the number of sperms and other accessory gland proteins necessary for effective fecundity and fertility evidently seen.

Figure 2c, 3c and table 1, 2 showed that female of $D$. malerkotliana mated with middle aged male had produced the greatest number of progeny (fertility) compare to old age and young males. Further second mated females with middle and young male had higher fertility rate, whereas first female that mated with old male produced highest progeny number than male mated with $2^{\text {nd }}$ female. These studies also suggest differentiates investment of male mated with $1^{\text {st }}$ and $2^{\text {nd }}$ females. Thus, these studies suggest that in D. malerkotliana male remating increases male fitness.

\section{Conclusion}

All the studies in D. malerkotliana suggests that male remating increases male fitness and males had inseminated and invested more in first female than with second female. Furthermore, middle aged male had a greater percentage of remaining and produces a greater number of progeny.

\section{Acknowledgements}

The authors are grateful to Professor and Chairman, Department of Studies in Zoology, University of Mysore, We are also grateful to the Drosophila Stock Centre, Dept. Of Zoology, University of Mysore for providing facilities to carry out this work.

\section{References}

[1] A. Ahuja, and R. S. Singh. "Variation and evolution of male sex combs in Drosophila: nature of selection response and theories of genetic variation for sexual Traits". Genetics 179: 503-509. 2008.

[2] T. D. Avent, Price T.A.R, and N. Wedell. "Age based female Preference in the fruit Fly Drosophila pseudoobscura". Animal Behaviour. 75 (4):1413-1421. 2008.

[3] I. R. Bock. "Taxonomy of the Drosophila bipectinata species complex". Univ. Texas Publ, 7103, 273-280. 1971.

[4] L. R. Bock, and M .R. Wheeler . "Drosophila melanogaster species group”. Univ. Tex. Publ.7213: 1-102. 1972.

[5] R. Brooks ,and D. J. Kemp. "Can older males deliver the good genes?”. Trends in Ecology and Evolution. 16:308-313. 2001 .

[6] P. Casares , M. C. Carracedo, B. Del Rio, R. Pinerio, L. GarciaFlorez, and A.R. Barros. "Disentangling the effects of mating propensity and mating choice in Drosophila".Evolution 52: 126-133. 1998.

[7] Cueva del Castillo, R. Nu'nez-Farfan, J. and Z. CanoSantana. "The role of body size in mating success of Sphenarium purpurascens in Central Mexico". Ecol. Entomol. 24, 146-155. 1999.

[8] F. Díaz-Fleischer, J. Arredondo, and M. Aluja. "Enriching early adult environment affects the copulation behaviour of a tephritid fly". Journal of Experimental Biology. 212:2120 2127. 2009.

[9] A. A. Elens, and J.M. Wattiaux. "Direct observation of sexual isolation”. Dros. Inf. Serv. 39:118-119. 1964.

[10] M.H. Gromko and D. W. Pyle. "Sperm competition, male fitness and repeated mating by female Drosophila melanogaster". Evolution 32: 588-593. 1978.

[11] M. H. Gromko, M.E.A. Newport, and M.C Kortier. "Sperm dependence of female receptivity to remating in Drosophila melanogaster". Evolution. 38:1273-1282. 1984.

[12] M. H. Gromko . "Genetic correlation of male and female mating frequency: evidence from Drosophila melanogaster" $r$. Anim. Behav. 43: 176-177. 1992.

[13] M. H Gromko, and T.A. Markow. "Courtship and remating in field populations of Drosophila". Anim. Behav. 45:253262. 1993.

[14] B. R. Guruprasad , S. N. Hegde, and M. S. Krishna. "Positive Relation between Male Size and Remating Success in Some Populations of Drosophila bipectinata" . Zoological Studies. 47(1): 75-83. 2008.

[15] T. F. Hansen, and D. K. Price. "Good genes and old age, do old mates provide superior genes?". Evolution biology, 8, 759-778. 1995.

[16] S. N. Hegde. "Studies on the cytotaxonomy and genetics of a few species of melanogaster species group of Drosophila". Thesis submitted to Univ, Mys. 1979.

[17] S. N. Hegde, and N. B. Krishnamurthy. "Studies on mating behaviour in the D. bipectinata complex", Aust. J. Zool. 27, 421-431. 1979. 
[18] S. N. Hegde , and M. S. Krishna. "Size assortative mating in Drosophila malerkotliana". Anim. Behav. 54:419-426. 1997.

[19] J. Hunt, R. Brooks, M. D. Jennions, M. J. Smith, C.L. Bentsen, and L. F. Bussiere, "High-quality male field crickets invest heavily in sexual display but die young". Nature 432, 1024-1027. 2004.

[20] A. P. Jha, and Rahman. "Cytogenetics of natural populations of Drosophila .I Role of chromosomal inversions in the evolution of the bipectinata species Complex", Chromosoma, 37, 445-454. 1972.

[21] S. Koref-Santibanez. "Effects of age and experience on mating activity in the sibling species of D. pavani and D. gaucha". Behav. Genet. 31, 287-297. 2001

[22] H. Kokko. "Good genes, old age and life-history trade-offs". Evolutionary Ecology. 12: 739-750. 1998.

[23] M.S. Krishna, and S. N. Hegde. Reproductive success of large and small flies in D.bipectinata complex. Current Science. 72: 747-750. 1997.

[24] S. Koref- Santibanez. "Effects of age and experience on mating activity in the sibling species Drosophila pavani and Drosophila gaucha”. Behavior Genetics, vol. 31, pp. $287-$ 297. 2001.

[25] LeBas, N. R., Hockman, L. R. and M.G. Ritchie. "Sexual selection in the gift-giving dance fly, Rhamphomyia sulcata, favors small males carrying small gifts". Evolution 58, 1763-1772. 2004.

[26] L. Levine , M. Asmussen, O. Olvera, J. R. Powell, M. E. Delarosa, V. M. Salceeda, M. I. Gaso, J. Gujman, and W. W. Anderson. "Population genetics of Mexican Drosophila. V. A. high rate of multiple inseminations in a natural population of Drosophila pseudoobscura". Am. Nat. vol. 116, pp. 493-503. 1980 .

[27] T. A. Markow, M. Quaid, S. Kerr. "Male mating experience and competitive courtship success in D. melanogaster". Nature. vol. 276, pp.821-822. 1978.

[28] K. P. Nicholas , F. G. Laura and A. Deborah, Roach. "Mating frequency and inclusive fitness in Drosophila melanogaster". The American naturalist, vol. 171, pp. 10-21. 2008.

[29] P. A. Parson. "Behavioral and ecological genetics: a study in Drosophila”. Oxford, UK: Clarenden Press. 1973.

[30] L. Partridge, M. Farquhar . "Sexual activity reduces lifespan of male fruit flies". Nature. 294:580-582. 1981.

[31] S. Pavkovic-lucic , and V. Kekic. "Influence of mating Experience on mating latency and copulation duration in Drosophila melanogaster females". Russian Journal of Genetics, vol. 45(7) pp. 875-877. 2009.

[32] S. Prakash . Association between mating speed and fertility in Drosophila robusta. Genetics. 57:655-663. 1967.
[33] M. Prathibha , M. S. Krishna and S. C. Jayaramu . "Male age influence on male reproductive performance in $D$. ananassae”. Italian Journal of Zoology, 78(2): 168173. 2011.

[34] J. A. Sanchez Prado, G. Blanco Lizana. "Mating patterns of different Adh genotypes of Drosophila melanogaster I. differences in mating ability". Genetica. 78:219-224.1989.

[35] B. N. Singh, Y. N. Dwivedi, and J. P. Gupta. "Sexual isolation among three species of the D. bipectinata species complex". Indian J. Exp.Biol,19, 898-900. 1981.

[36] B. N. Singh, and S. Chatterjee. "Variation in mating propensity and fertility in isofemale strains of Drosophila ananassae". Genetica 73: 237. 1987.

[37] R. S. Singh. "Broad-sense sexual selection, sex gene pool evolution, and speciation". Genome.42:1033-41.1999.

[38] S. R. Singh, and B. N. Singh. "Male remating in Drosophila ananassae: evidence for interstrain variation in remating time and shorter duration of copulation during second mating”. Zool. Sci. 17: 389-393. 2000.

[39] S. R. Singh, B. N. Singh and H. F. Hoenigsberg "Female remating, sperm competition and sexual selection in Drosophila". Genetics and Molecular Research, vol. 1(3), pp. 178-215. 2002

[40] S. R. Singh, and B. N. Singh, "Female remating in Drosophila: comparison of duration of copulation between first and second mating in six species". Current Scince, vol. 86(3) pp. 465-470. 2004

[41] S. Sisodia , B. N. Singh. "Size dependent sexual selection in Drosophila ananassae". Genetica 121: 207-21. 2004.

[42] K. Somashekar , and M. S. Krishna. "Evidence of female preference for older males in D. bipectinata". Zoological studies, 50(1): 1-15. 2011.

[43] E. B. Spiess. "Mating Propensity and Its Genetic Basis in Drosophila" Essays in Evolution and Genetics in Honor of Theodosius Dobzhansky, Hecht, M. K. and Sreere, W .C ., Eds., Amsterdam. pp. 315-379. 1970.

[44] M. E. Turner, and W. A. Anderson. "Multiple mating and female fitness in Drosophila melanogaster". Evolution, vol. 37, pp. 714-723. 1983.

[45] S. Y. Yang, L. L. Wheeler, and I. R. Bock. Isozyme variations phylogenetic relationships in the $D$. bipectinata species complex. Univ. Texas Publ, 7213, 213- 227. 1972.

[46] M. Zuk. "Parasite load, body size, and age wild-caught male field crickets (orthoptera Gryllidae) effects on sexual selection”. Evolution. 42:969-976. 1988. 